\title{
Mortalidade feminina na região Sul do Município de São Paulo: qualidade da certificação médica dos óbitos
}

\section{Maternal mortality in Brazil: quality of medical certification of deaths}

Arnaldo Augusto Franco de Siqueira, Evani Marzagão Beringhs Rio, Ana Cristina d'Andretta Tanaka, Néia Schor, Augusta Thereza de Alvarenga e Ludmila Chaves Almeida**

Departamento de Saúde Materno-Infantil da Faculdade de Saúde Pública da Universidade de São Paulo. São Paulo, SP - Brasil 


\title{
Mortalidade feminina na região Sul do Município de São Paulo: qualidade da certificação médica dos óbitos* Maternal mortality in Brazil: quality of medical certification of deaths
}

\author{
Arnaldo Augusto Franco de Siqueira, Evani Marzagão Beringhs Rio, Ana Cristina \\ d'Andretta Tanaka, Néia Schor, Augusta Thereza de Alvarenga e Ludmila Chaves \\ Almeida**
}

Departamento de Saúde M aterno-Infantil da Faculdade de Saúde Pública da U niversidade de São Paulo. São Paulo, SP - Brasil

\section{Descritores}

Atestados de óbito.

Causa básica de morte.

Classificação

Internacional de Doen-

ças. Validade.

\author{
Keywords \\ Death certificates. \\ Underlying cause of \\ death. International \\ Classification of Dis- \\ eases. Validity.
}

\author{
Resumo \\ Objetivo \\ Avaliar a qualidade da certificação médica dos óbitos de mulheres de 10 a 49 anos na \\ região Sul do Município de São Paulo. \\ Métodos \\ Utilizou-se a metodologia Puffer para investigar as causas de óbito de todas as \\ mulheres de 10 a 49 anos residentes na região e falecidas no ano de 1989 (num total \\ de 664 óbitos). Foram comparadas as causas de morte relatadas nos atestados de \\ óbitos originais com as refeitas. Calculou-se o grau de sensibilidade das causas e o \\ índice de kappa. \\ Resultados
}

Obteve-se alta sensibilidade entre as declarações de óbito originais e corrigidas nos grupos de causas (CID-9) das "Doenças do Aparelho Circulatório" (91,9\%), "Neoplasias" $(89,7 \%)$ e "Causas Externas" $(84,1 \%)$. Houve outros com sensibilidades muito baixas. Desses, chama a atenção o capítulo "Transtornos Mentais" $(34,3 \%)$ que, de 11, passa a ter 32 óbitos em que, na maioria dos casos, não havia sido feito o diagnóstico de alcoolismo. Também o capítulo "Complicações da Gravidez, Parto e Puerpério" apresentou uma sensibilidade baixa (44,9\%). O índice de kappa foi de 0,63 , o que indica uma concordância total regular.

Discussão

Ainda é insatisfatória a qualidade da certificação médica dos óbitos na área estudada. Essa má qualidade pode afetar negativamente as propostas de intervenção, tornando pouco visíveis problemas tão sérios para a atenção à saúde da mulher.

\author{
Abstract \\ Objective \\ To evaluate the quality of the medical certification of deaths of 10-49 year-old \\ women, resident in the Southern region of the city of S. Paulo \\ Methods \\ The Puffer methodology was utilized to investigate the causes of death of all 10-49 \\ year-old women, resident in the region, and deceased in the year 1989 (664 deaths
}

Correspondência para/Correspondence to:

A. A. Franco de Siqueira

Av. Dr. Arnaldo, 715

01246-904 São Paulo, SP - Brasil

E-mail: aafsique@usp.br
* Subvencionado pela Fundação de Amparo à Pesquisa do Estado de São Paulo

(Fapesp) Processo 88/2368-1.

**Bolsista CN Pq/PIBIC/U SP

Edição subvencionada pela Fapesp (Processo n 98/13915-5).

Recebido em 23.10.1998. Reapresentado em 28.5.1999. Aprovado em 1.6.1999. 
in the total). The main causes of death in the original death certificates and the "new" causes of death arisen from the research were compared. The sensitivity and the kappa index were calculated.

Results

In some chapters of the International Classification of Diseases and Causes of Death, 9th Revision (CID-9), a high sensitivity was found: "Diseases of the Circulatory System" (91.9\%), "Neoplasms" (89.7\%) and "External Causes" (84.1\%). In some others, a very low sensitivity was found. The chapter "Mental Illnesses", with a 34.3 percent sensitivity only, must be mentioned. From 11 deaths originally classified in this chapter, 32 cases were found. In most of these "new" cases, the main cause of death was found to be alcoholism. The chapter "Complications of Pregnancy, Delivery and the Puerperium", also showed a low sensitivity (44.9\%). The kappa index was found to be 0.63 , which indicated a regular concordance.

Discussion

The quality of the medical certification of causes of death is still unsatisfactory in the studied area. This poor quality may affect negatively the interventions in the area of women's health, masking the severity of important problems.

\section{INTRO DUÇÃO}

Os estudos de mortalidade continuam sendo importante instrumento para o monitoramento das condições de saúde da população e para o planejamento, implementação e avaliação das ações de saúde. Sua utilidade, entretanto, depende da fidedignidade das suas fontes de informação.

A qualidade e confiabilidade das declarações de óbitos vêm sendo examinadas e questionadas em diversos países, bem como no Brasil. Milanesi e Laurenti ${ }^{6}$ já demonstraram esta preocupação, em que salientam a precariedade no preenchimento dos atestados de óbito. Fonseca e Laurenti ${ }^{3}$ confirmam este mesmo resultado. Entretanto, Laurenti et al. ${ }^{5}$ referem ter encontrado boa qualidade nas informações contidas nas declarações de óbito para o Município de São Paulo.

$\mathrm{Na}$ literatura internacional Hunt et al. ${ }^{4}$ verificaram a acurácia dos atestados de óbito em pacientes asmáticos em um estudo de coorte entre 1964 e 1983 em Rochester, e relataram os mesmos problemas encontrados em trabalhos nacionais: 1. deficiência no preenchimento dos certificados por falta de treinamento dos médicos; 2. dificuldade de obtenção de dados de autópsia que deveriam completar os dados contidos no atestado; 3. deficiência na determinação da causa básica de morte?

No presente estudo o objetivo foi realizar uma comparação entre a causa básica de óbito contida nos atestados originais e refeitos, após coleta de uma série de informações complementares junto aos familiares das mulheres falecidas, hospitais onde foram atendidas, bem como dados de autópsia, verificando o grau de concordância das mesmas e as principais diferenças encontradas.

\section{MÉTO DOS}

Foram selecionadas todas as declarações de óbitos de mulheres com idade de 10 a 49 anos, ocorridos no ano de 1989, residentes nesta região, num total de 664 óbitos. Esta área é a mais extensa do Município de São Paulo, correspondendo a 42\%, com uma população aproximada de 1.600 .000 habitantes (censo de 1991), distribuídos de forma heterogênea.

O estudo compreendeu três etapas, à semelhança do delineamento de pesquisa proposto por Puffer ${ }^{9}$. Na primeira etapa foi aplicado um formulário aos familiares das mulheres falecidas, contendo variáveis de natureza clínica, epidemiológica, demográfica, social e de condições e qualidade da assistência à saúde recebida. Na segunda, foram feitas visitas aos hospitais para coleta de dados de prontuários médicos. A terceira etapa foi relacionada à obtenção de laudos de autópsia junto ao Instituto Médico Legal (IML) e aos Serviços de Verificação de Óbitos (SVO).

A seguir, foram revisados todos os protocolos criados a partir dos atestados de óbito. A codificação diagnóstica foi refeita quando necessário, utilizando-se a Classificação Internacional de Doenças - 9a revisão (CID-9) ${ }^{8}$.

Para a determinação da causa básica de morte tanto nos atestados originais, como nos refeitos, utilizou-se o Sistema de Seleção de Causa Básica (SCB) para uso em microcomputadores ${ }^{10}$.

A partir daí, todas as causas de óbito foram classificadas segundo os capítulos da CID-9 para os atestados originais e os refeitos, separadamente, possibilitando uma comparação entre eles.

No presente trabalho, para a análise estatística desses dados, foram utilizadas a frequiência simples, percentagens e as 
medidas de associação: grau de sensibilidade e teste de Kappa. Por grau de sensibilidade foi considerado o percentual de óbitos por um determinado grupo de causas, classificados corretamente nos atestados originais. Para interpretação da estatística Kappa, foram utilizados os critérios de Landis e Koch, citados por Fleiss²:

$$
\begin{aligned}
& \mathrm{K}>0,75=\text { excelente concordância } \\
& 0,40>\mathrm{K}<0,75=\text { regular a boa concordância } \\
& \mathrm{K}<0,40=\text { concordância fraca }
\end{aligned}
$$

\section{RESU LTADOS E DISCUSSÃO}

A Tabela 1 mostra a distribuição das causas básicas de óbito, nos atestados originais e refeitos, de acordo com os capítulos da CID-9. Os números que aparecem na diagonal mostram a concordância, isto é, os casos em que, no atestado original e refeito, atribuiu-se uma causa básica pertencente ao mesmo capítulo.

O cálculo do teste de kappa para avaliar a concordância entre os atestados originais e os refeitos alcançou o valor de 0,63 . Isto significa, de acordo com os critérios já citados, que os atestados originais, no seu conjunto, não revelam uma boa concordância com os refeitos, levando a informações distorcidas sobre as reais causas de morte, podendo resultar em intervenções menos adequadas.

Para localizar os capítulos em que houve maior ou menor concordância, foi utilizada a sensibilidade, cujos resultados podem ser vistos na Tabela 2 .

O grau de sensibilidade variou bastante. As maiores proporções ocorreram nos capítulos: VII- "Doenças do Aparelho Circulatório" (91,9\%), II- "Neoplasias" $(89,7 \%)$ e CE- "Causas Externas” (84,1\%).

As sensibilidades mais baixas foram verificadas nos capítulos: V- "Transtornos Mentais" (34,3\%), XI"Complicações da Gravidez Parto e Puerpério" (44,9\%), 14-“Anomalias Congênitas” (50,0\%), 13 "Doenças do Sistema Ósteo Muscular e Tecido Conjuntivo" (55,6\%). Ainda apresentaram sensibilidade baixa os capítulos referentes a "Sintomas, Sinais e Afecções Maldefinidas" (57,1\%) e "Doenças Infecciosas e Parasitárias" $(65,4 \%)$.

O significado e a razão desses resultados com baixa sensibilidade têm conseqüências distintas para o

\begin{tabular}{|c|c|c|c|c|c|c|c|c|c|c|c|c|c|c|c|c|}
\hline \multirow{2}{*}{$\begin{array}{l}\text { Capítulos } \\
\text { do atestado } \\
\text { de óbito } \\
\text { corrigido }\end{array}$} & \multicolumn{16}{|c|}{ Capítulos do atestado de óbito original } \\
\hline & 1 & ॥ & III & IV & v & VI & VII & VIII & IX & $x$ & $X I$ & XIII & XIV & $\mathrm{XVI}$ & CE & Total \\
\hline 1 & 34 & - & - & - & - & - & 12 & 2 & 1 & - & 1 & - & - & 2 & - & 52 \\
\hline II & 2 & 96 & 2 & - & - & - & 3 & 1 & - & 1 & - & - & - & 1 & 1 & 107 \\
\hline III & - & - & 19 & - & - & - & 1 & 5 & - & 2 & - & - & - & - & - & 27 \\
\hline IV & - & - & - & - & - & - & 1 & 1 & - & - & - & - & - & - & - & 2 \\
\hline V & - & - & - & - & 11 & 1 & 8 & 4 & 6 & 1 & - & - & - & - & 1 & 32 \\
\hline VI & - & - & - & - & - & 6 & - & 1 & - & - & - & - & - & - & - & 7 \\
\hline VII & - & - & 1 & - & - & 2 & 136 & 1 & 2 & 3 & - & - & - & 3 & - & 148 \\
\hline VIII & - & - & - & - & - & 1 & 3 & 37 & - & 2 & - & - & - & 1 & - & 44 \\
\hline IX & 1 & - & - & - & - & - & 2 & - & 19 & - & - & - & - & - & - & 22 \\
\hline$x$ & 2 & - & - & - & - & - & 2 & - & 1 & 10 & - & - & - & - & - & 15 \\
\hline XI & - & 1 & 1 & 1 & - & - & 3 & 7 & 1 & 5 & 22 & 1 & 1 & 3 & 3 & 49 \\
\hline XIII & - & - & - & 1 & - & 1 & 1 & - & - & - & - & 5 & - & 1 & - & 9 \\
\hline XIV & - & - & - & - & - & - & - & 1 & 1 & - & - & - & 2 & - & - & 4 \\
\hline XVI & - & - & - & - & - & 1 & 3 & 2 & - & - & - & - & - & 8 & - & 14 \\
\hline CE & 2 & 1 & - & - & - & 3 & 3 & 7 & 2 & - & - & - & - & 3 & 111 & 132 \\
\hline Total & 41 & 98 & 23 & 2 & 11 & 15 & 178 & 69 & 33 & 24 & 23 & 6 & 3 & 22 & 116 & 664 \\
\hline
\end{tabular}

Tabela 1 - Causa básica de morte em mulheres de 10 a 49 anos, comparando-se os atestados de óbito originais e os corrigidos após pesquisa empírica. Município de São Paulo, 1989.*

* Ver denominação dos capítulos na Tabela 2. 
Tabela 2 - Causas de morte em mulheres de 10 a 49 anos, casos em que houve concordância entre atestados originais e refeitos, e o grau de sensibilidade. Município de São Paulo, 1989.

\begin{tabular}{|c|c|c|c|c|c|c|}
\hline \multirow{2}{*}{$\begin{array}{l}\text { Capítulos Cid -9 } \\
\text { I-D. Infec. Parasitária }\end{array}$} & \multicolumn{2}{|c|}{$\begin{array}{l}\text { Atestado original } \\
\mathbf{N} 0 \quad \text { Posição }\end{array}$} & \multicolumn{2}{|c|}{$\begin{array}{l}\text { Atestado refeito } \\
\text { No } \quad \text { Posição }\end{array}$} & \multirow{2}{*}{$\begin{array}{c}\begin{array}{c}\text { Casos em que } \\
\text { houve concordância }\end{array} \\
34\end{array}$} & \multirow{2}{*}{$\begin{array}{c}\text { Sensibilidade } \\
65,4\end{array}$} \\
\hline & 41 & 5 & 52 & 4 & & \\
\hline $\begin{array}{l}\text { II-Neoplasia } \\
\text { III-D. End-N ut e }\end{array}$ & 98 & 3 & 107 & 3 & 96 & 89,7 \\
\hline Transtornos Imun. & 23 & 8 & 27 & 8 & 19 & 70,3 \\
\hline IV- Sangue O rg. Hemat. & 2 & 14 & 2 & 15 & - & - \\
\hline V- Transtornos M entais & 11 & 11 & 32 & 7 & 11 & 34,3 \\
\hline VI- Sistema N ervoso & 15 & 10 & 7 & 13 & 6 & 85,7 \\
\hline VII- Ap.Circulatório & 178 & 1 & 148 & 1 & 136 & 91,9 \\
\hline VIII- Ap. Respiratório & 69 & 4 & 44 & 6 & 37 & 84,1 \\
\hline IX-Ap. Digestivo & 33 & 6 & 22 & 9 & 19 & 86,4 \\
\hline X- Ap. Geniturinário & 24 & 7 & 15 & 10 & 10 & 66,7 \\
\hline XI- Grav. Part, Puerp. & 23 & 8 & 49 & 5 & 22 & 44,9 \\
\hline XIII-Sist. O steomusc. & 6 & 12 & 9 & 12 & 5 & 55,6 \\
\hline XIV- Ano. Congênitas & 3 & 13 & 4 & 14 & 2 & 50,0 \\
\hline XVI- Maldefinido & 22 & 9 & 14 & 11 & 8 & 57,1 \\
\hline CE- Causas Externas & 116 & 2 & 132 & 2 & 111 & 84,1 \\
\hline Total & 664 & - & 664 & - & - & - \\
\hline
\end{tabular}

sistema de saúde. Como, por exemplo, no capítulo de "Sintomas, Sinais e Afecções Maldefinidas", em que a sensibilidade baixa é considerada um fator negativo. Observa-se com as informações adicionais do estudo que, embora tenham sido encontrados 22 casos, este número poderia ser menor, 14 casos, conforme o resultado final.

As três primeiras causas de morte, além de mostrarem uma sensibilidade boa, permaneceram na mesma posição após a correção. Pela ordem: $1^{\mathrm{a}}$ - Doenças do Aparelho Circulatório, $2^{\mathrm{a}}$ - Causas Externas e $3^{\mathrm{a}}$ Neoplasias.

\section{Análise das Alterações por Capítulos}

O capítulo V: "Transtornos Mentais" passou da $11^{a}$ para a $7^{a}$ posição após revisão dos atestados, com um grau de sensibilidade bastante baixo $(34,3 \%)$.

- 6 casos vieram do capítulo VII "Doenças do Aparelho Circulatório".

- 3 casos do capítulo VIII "Doenças do Aparelho Respiratório".
- 6 casos do capítulo IX "Doenças do Aparelho Digestivo".

Eram todos casos de alcoolismo, não especificados, que haviam sido classificados em outros capítulos, sem esta menção.

Este resultado traz importantes implicações para a assistência à saúde da mulher. $\mathrm{O}$ alcoolismo tem sido visto como uma questão mais ligada ao sexo masculino e aqui vemos que, após correção, ele passa a ser uma importante causa de mortalidade feminina, alcançando aproximadamente $4,5 \%$ do total de mortes.

Assim, o problema do alcoolismo está deixando de figurar com a relevância que merece na agenda da assistência à mulher devido ao fato de que não aparece com a real magnitude nas estatísticas oficiais.

O capítulo XI: "Complicações da Gravidez, do Parto e do Puerpério" passa da $8^{\mathrm{a}}$ para a $5^{\mathrm{a}}$ posição, na ordem de ocorrências, apresentando um baixo grau de sensibilidade $(44,9 \%)$. Dos 23 casos inicialmente relatados, obtiveram-se 49 após revisão. Entretanto, dos casos considerados como morte materna nas declarações originais, apenas um deixou de ser assim 
classificado: tratava-se de uma mulher que estava no final do puerpério, cuja morte foi devida a uma miocardite chagásica, sem relação com a gravidez, passando então para o capítulo I: "Doenças Infeciosas e Parasitárias".

Os 27 casos considerados como morte materna após revisão estavam codificados nos atestados originais da seguinte forma:

- 7 casos pertencentes ao capítulo de "Doenças do Aparelho Respiratório" : um caso de asma considerado agravado pela presença da gravidez não relatada e os demais codificados como insuficiência respiratória aguda e broncopneumonia eram, na realidade, diagnósticos terminais de complicações da gravidez, parto e puerpério.

- 5 casos classificados anteriormente no capítulo de "Doenças do Aparelho Geniturinário" eram casos de aborto e infecção puerperal.

- 3 casos considerados como "Afecções Maldefinidas" foram esclarecidos pela investigação; também se tratava de casos de aborto.

- 3 casos estavam codificados como pertencentes ao capítulo do "Aparelho Circulatório"; eram cardiopatias descompensadas pela gravidez não relatada e uma hemorragia meningea decorrente de uma eclâmpsia grave.

- 3 casos vieram do capítulo de "Causas Externas": um deles, codificado como acidente, na revisão mostrou ser um caso de mola hidatiforme. Devido à grande divergência, questionou-se a possibilidade de ter havido uma troca de atestado. Outro caso, considerado como acidente anestésico sem outra menção, na realidade, ocorreu durante uma cesárea sem indicação precisa. $\mathrm{O}$ terceiro caso codificado como hemorragia externa traumática era mais um caso de aborto.

- 6 causas restantes vieram de seis outros capítulos, estando classificadas como: neoplasia, diabetes, coagulação intravascular disseminada, doença crônica do fígado, artrite reumatóide e cardiopatia congênita, em que a presença da gravidez não relatada interferiu na conduta e na evolução do quadro.

Deve-se considerar, no entanto, que para cada um dos 22 casos mantidos nos atestados originais e refeitos foram aqui classificados outros 1,22. Ou seja, $122 \%$ a mais de mortes maternas. Estes dados confirmam resultados de outros autores como, por exemplo, Laurenti et al. ${ }^{15}$ que encontraram, em 1986, 1,24 novos óbitos maternos para cada caso "oficial". Assim a subinformação do óbito materno aqui é como em outros países (mesmo os desenvolvidos) conforme o M.M.W.R. ${ }^{1}$, muito importante, levando também à subestimação de um tão grande problema de saúde como é a assistência no ciclo gravídico puerperal.

O capítulo XIII: "Doenças do Sistema Ósteo Muscular e Tecido Conjuntivo" apresentou um pequeno número de casos, permanecendo na mesma posição, mas com baixa sensibilidade. Nota-se, entretanto, que dos 6 casos iniciais, houve concordância em 5 e, ao final, foram encontrados 9 casos vindos de vários outros capítulos. $\mathrm{O}$ caso discordante diagnosticava a causa básica do óbito como artrite reumatóide, tendo sido constatado, no estudo, que se tratava de mais um caso de aborto.

Assim, importantes problemas, como a participação do aborto como causa de morte materna, também ficam camuflados devido à deficiente certificação médica, ou talvez às dificuldades decorrentes da legislação vigente.

O capítulo X: “Aparelho Geniturinário" também merece consideração devido à baixa sensibilidade encontrada (66,7\%). Da sétima posição ele cai para a décima. Dos 24 casos iniciais houve concordância em apenas 10, tendo ficado reduzido a 15 casos após correção. Foram transferidos 5 casos para o capítulo XI "Complicações da Gravidez Parto e Puerpério", como descrito anteriormente, ficando os demais distribuídos em vários outros capítulos.

No capítulo III: "Doenças das Glândulas Endócrinas, da Nutrição e do Metabolismo e Transtornos Imunitários", observou-se uma sensibilidade regular (70,3\%). Houve concordância em 19 dos 23 casos relatados inicialmente, chegando-se ao final com 27 casos. As maiores alterações foram:

- 6 casos de diabetes mellitus. Constava nos atestados originais apenas a causa terminal como: broncopneumonia ou insuficiência respiratória (5 casos do capítulo VIII) ou ainda como outras doenças inespecíficas: cardiomiopatia ( 1 caso do capítulo VII), sem a especificação da existência do diabetes.

- 2 casos de Aids que tinham sido classificados como outras doenças inespecíficas (nefropatia) do capítulo X. 
No capítulo I: "Doenças Infecciosas e Parasitárias", a sensibilidade foi considerada baixa $(65,4 \%)$. Dos 41 casos obtidos nos atestados de óbitos originais, houve concordância em apenas 34, obtendo-se, entretanto, 52 casos após correção dos atestados.

- 12 casos vieram do capítulo de "Doenças do Aparelho Circulatório". Estavam codificados como miocardiopatias inespecíficas, que, após investigação, foram definidas como chagásicas, passando então para o capítulo de "Doenças Infecciosas e Parasitárias".

- 2 casos codificados como pertencentes às "Doenças do Aparelho Respiratório", após análise, aparecem como septicemia.

- 1 caso que estava no capítulo de "Doenças do Aparelho Digestivo" era esquistossomose, passando então para o capítulo I.

- 1 caso que veio do capítulo de "Complicações da Gravidez, do Parto e do Puerpério": tratava-se de uma puérpera que morreu devido a uma miocardiopatia chagásica, não tendo sido evidenciada qualquer influência da gravidez.

- 2 casos foram esclarecidos no capítulo XVI: “Afecções Maldefinidas" como sendo casos de miocardiopatia chagásica e septicemia.

- 7 casos que haviam sido codificados como pertencentes ao capítulo I passaram para os seguintes capítulos: 2 casos para o capítulo de "Neoplasias", 1 para o de "Doenças do Aparelho Digestivo", 2 para o de "Doenças do Aparelho Geniturinário" e 2 para o capítulo de "Causas Externas".

Esses dados mostram que as doenças infecciosas e parasitárias são ainda importantes causas de morte no Brasil, estando entre as cinco primeiras, indicando a importância de políticas de saúde nesta área. Deve-se lembrar que a Aids não estava até então classificada nesse capítulo e ainda era um problema de saúde menos importante entre as mulheres, fato este muito diferente do que ocorre nos dias atuais.

\section{REFERÊNCIAS}

1. Centers for Disease Control and Prevention. Maternal mortality: Pilot surveillance in seven states. M MWR 1985;34:7011.

2. Fleiss JL. Statistical methods for rates and proportions. 2nd ed. New York: John Wiley Sons; 1981.

3. Fonseca LAM, Laurenti RA. A qualidade da certificação médica da causa de morte em São Paulo, Brasil. Rev Saúde Pública 1974;8:21-9.

4. Hunt LW, Silverstein MD, Reed CE, O'Conell E J, O' Fallon WM, Yunfinger JW. Accuracy of the death certificate in a population - based study of asthmatic patients. J Am Medic Assoc 1993;269:1947-52.

5. Laurenti R, Buchalla CM, Lolio CA, Santo AH, Jorge MHPM. Mortalidade de mulheres em idade fértil no Município de São Paulo (Brasil), 1986. I- Metodologia e Resultados Gerais. Rev Saúde Pública 1990; 24:128-33.
6. Milanese ML, Laurenti R. 0 estudo interamericano de mortalidade em São Paulo. I- Estado atual da certificação médica da causa de óbito do distrito da capital. Rev Assoc Med Bras 1964;10:111-6.

7. Nobre LC, Victora CG, Barros FC, Lombardi C, Teixeira AMB, Ficha SC. Avaliação da qualidade da informação sobre a causa básica de óbitos infantis no Rio Grande do Sul (Brasil). Rev Saúde Pública 1989:23:207-13.

8. O rganização Mundial da Saúde. Manual da classificação estatística internacional de doenças, lesões e causas de óbito, 9a Rev. São Paulo: Centro da OMS para Classificação de Doenças em Português; 1985.

9. Puffer RR, G riffith GW. Características de la mortalidad urbana. Washington (DC): O rganización Panamericana de la Salud; 1968. (OPS - Publicación Científica, 151).

10. Santo $A H$, Pinheiro CE. U so do computador na seleção da causa básica de morte. Bol O ficina Sanit Panam 1995;119:319-27. 